\title{
Recognizing the limited applicability of Job plots in studying host-guest interactions in supramolecular chemistry
}

Filip Ulatowski, Kajetan Dąbrowa, Tomasz Bałakier, and Janusz Jurczak

Institute of Organic Chemistry, Polish Academy of Sciences, Kasprzaka 44/50 01-224 Warsaw, Poland

E-mail: janusz.jurczak@icho.edu.pl

\section{Electronic Supplementary Info}

\section{Table of Contents}

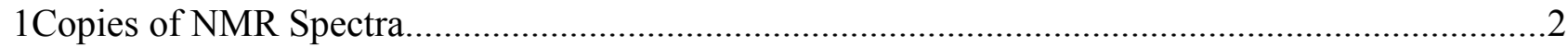

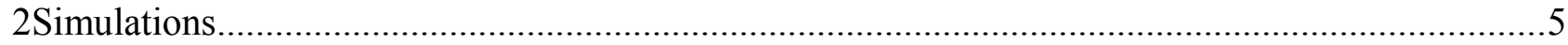

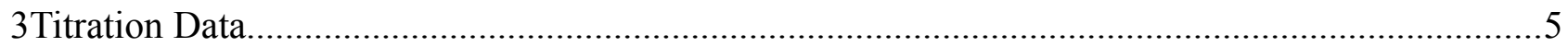




\section{Copies of NMR Spectra}
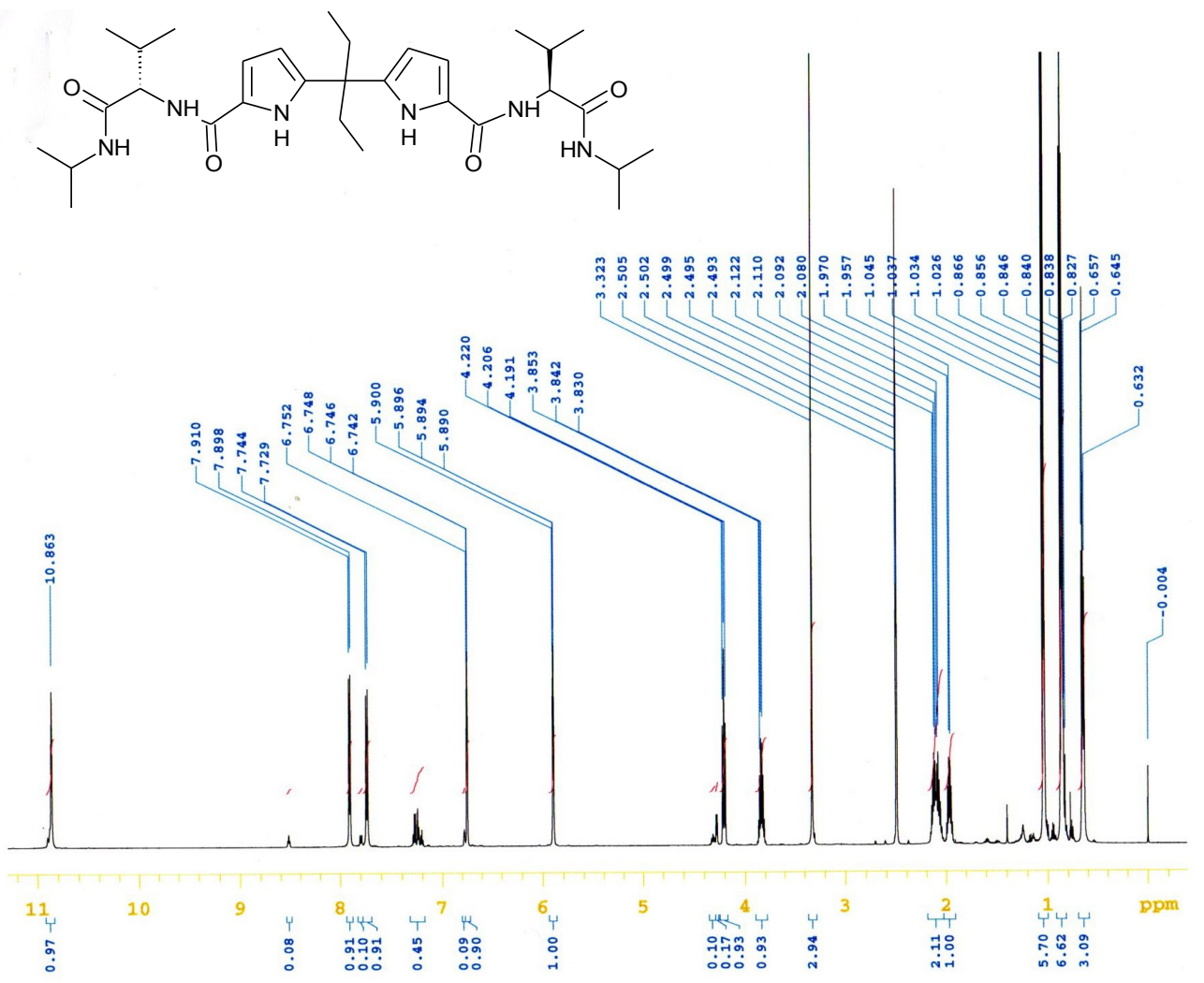

Fig. $1{ }^{1} \mathrm{H}$ NMR spectrum of $\mathbf{1}$

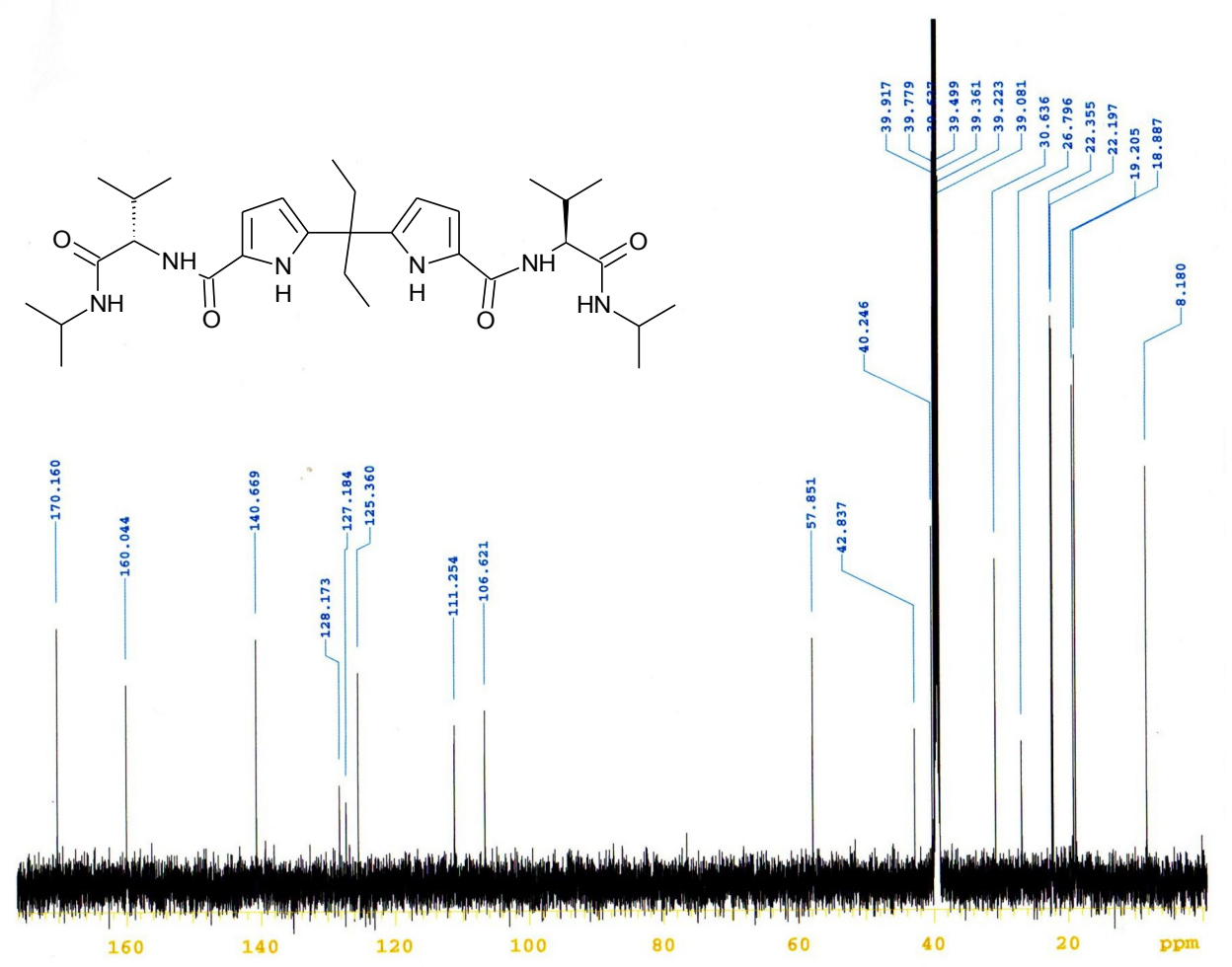

Fig. $2{ }^{13} \mathrm{C}$ NMR spectrum of $\mathbf{1}$ 


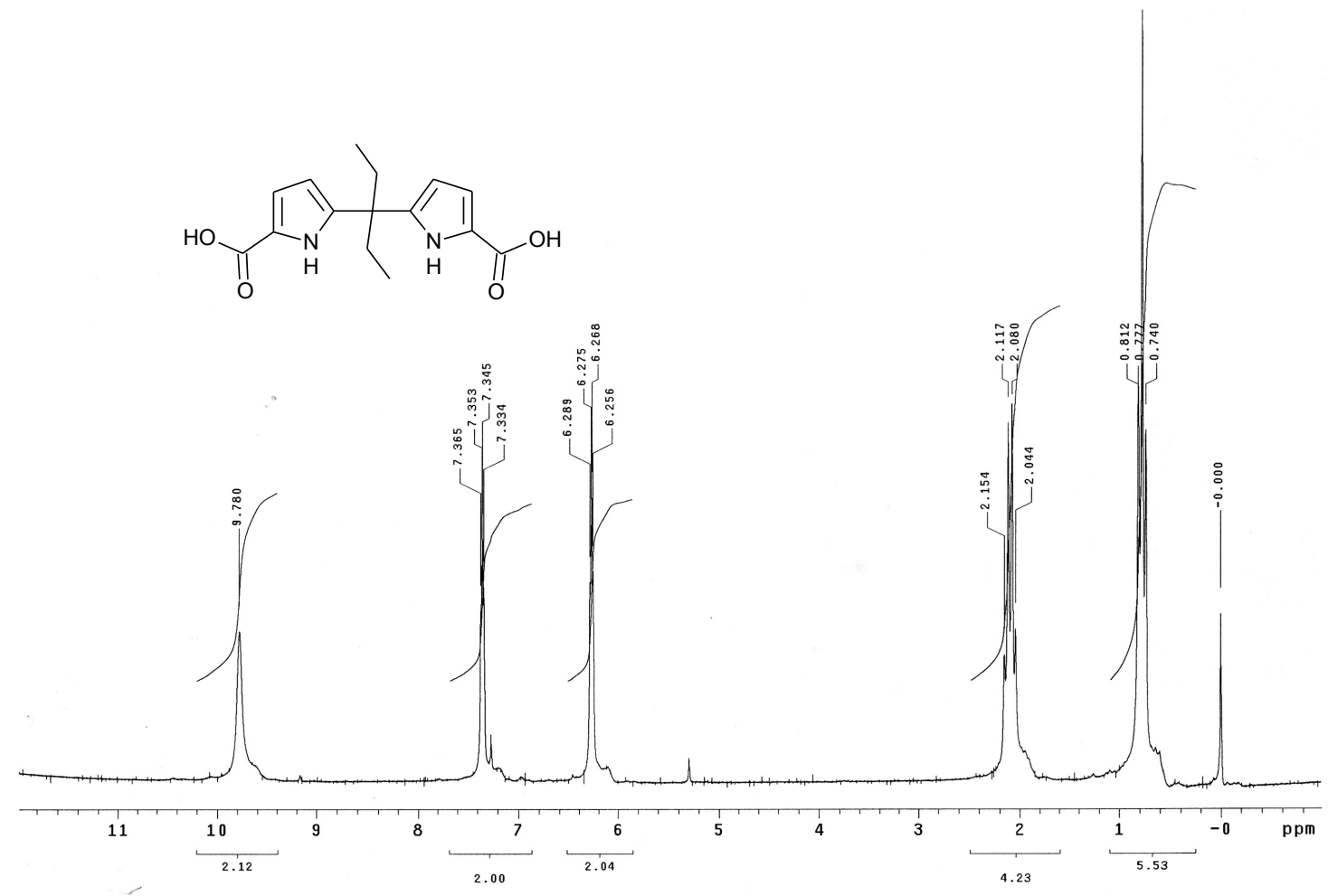

Fig. $3{ }^{1} \mathrm{H}$ NMR spectrum of $\mathbf{8}$

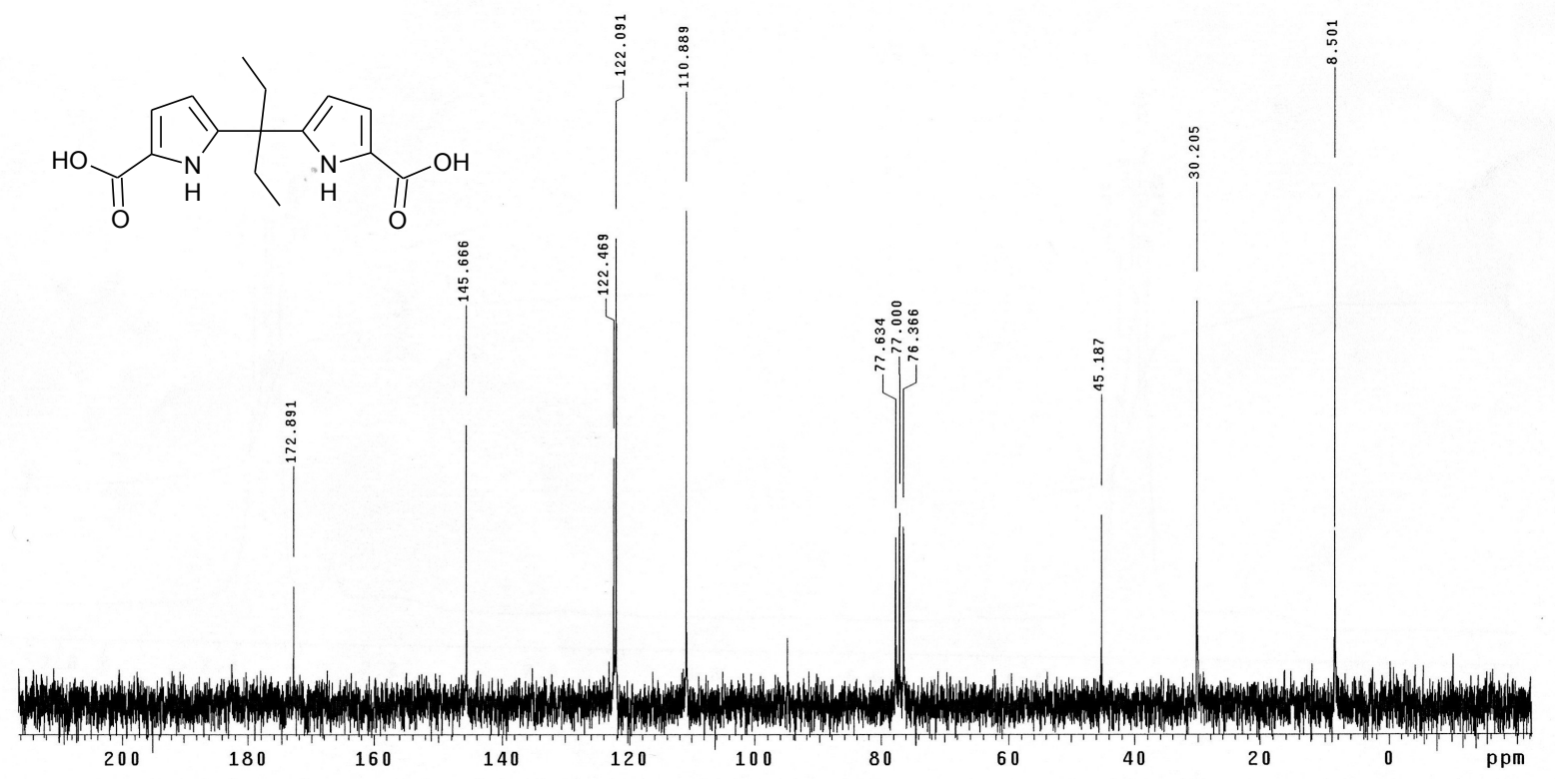

Fig. $4{ }^{13} \mathrm{C}$ NMR spectrum of $\mathbf{8}$ 


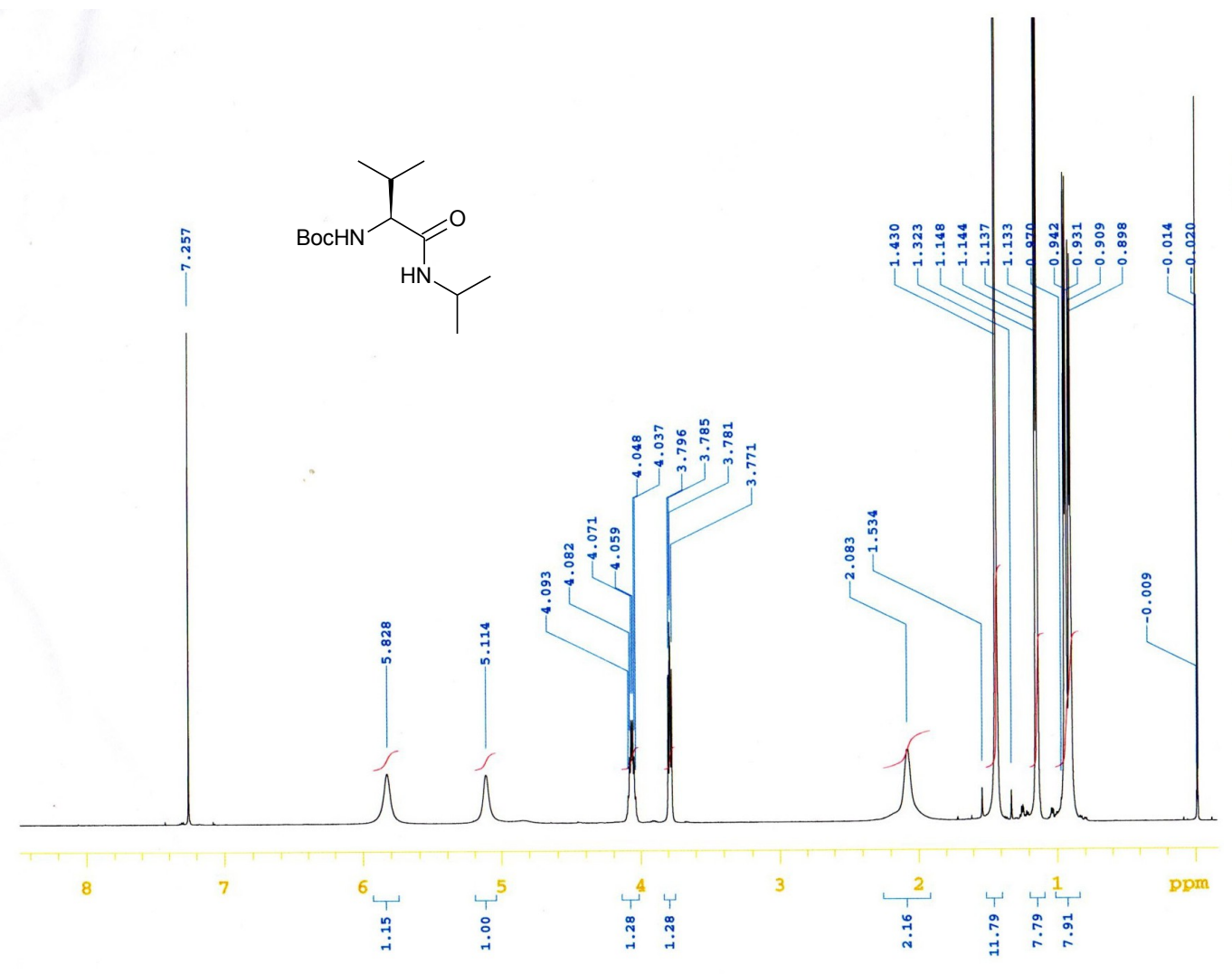

Fig. $5{ }^{1} \mathrm{H}$ NMR spectrum of $\mathbf{1 0}$

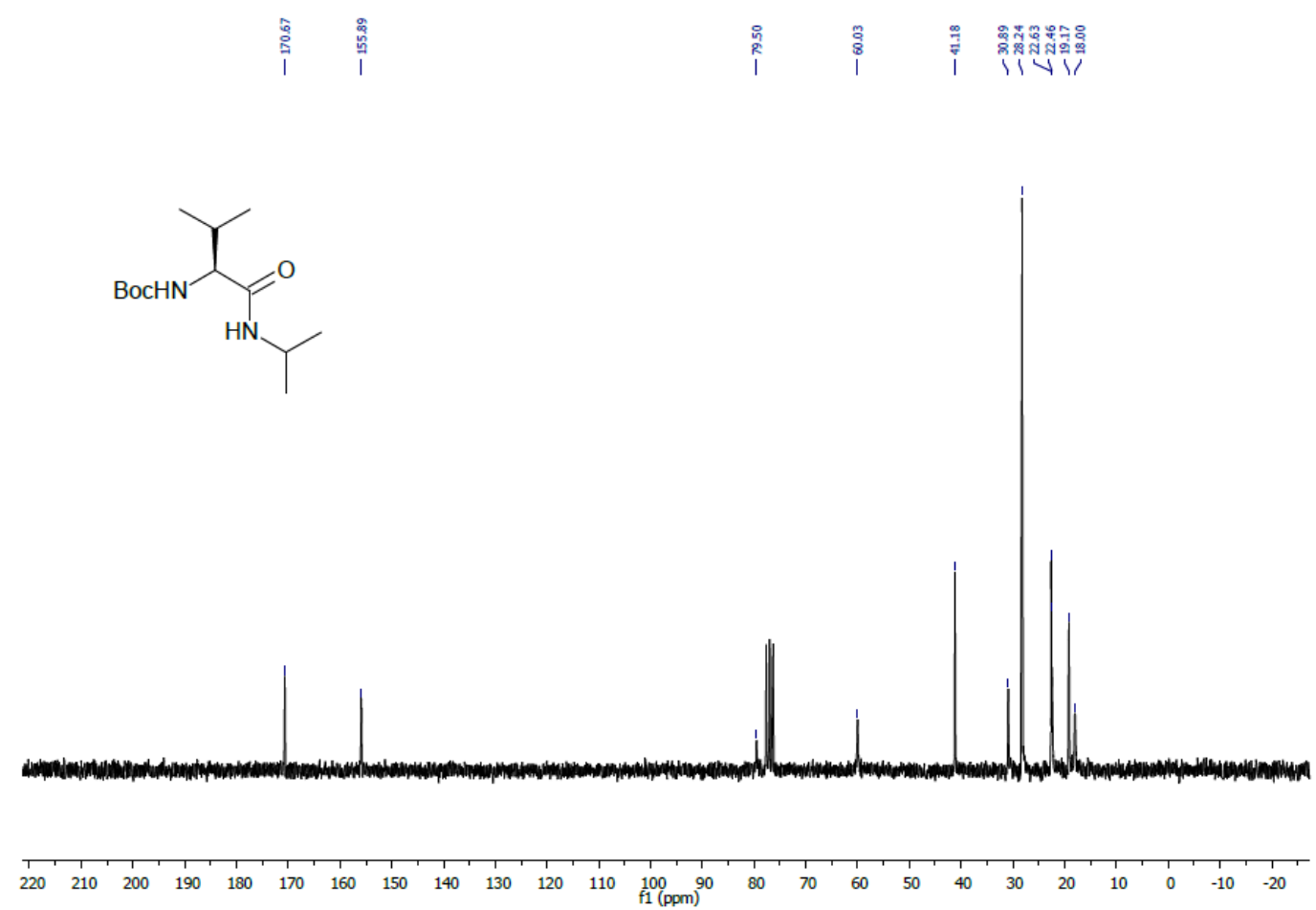

Fig. $6{ }^{13} \mathrm{C}$ NMR spectrum of $\mathbf{1 0}$ 


\section{Simulations}

The simulated data for Job plots and titrations were obtained in an OpenOfficeCalc spreadsheet. The cubic equation describing the binding isoterms for $\mathrm{HG}+\mathrm{HG}_{2}$ model was solved analytically using Cardano method with Vieta's substitution. The spreadsheet is available from the authors.

When simulating the titration datapoints for graphs in Table 4 the number of equivaletns of guest was in each case adjusted to reach a plateau: the difference between $Y$ in the last two titration points is less then $1 \%$ of total $\Delta Y:\left(Y_{30}-Y_{29}\right)<0.01 *\left(Y_{30}-Y_{0}\right)$. The simulated data were then fitted with 1:1 model using WinEqNMR software.

\section{Titration Data}

\begin{tabular}{|c|c|c|c|}
\hline & $\begin{array}{l}\text { Host: } \\
\text { Guest: } \\
\text { Method: } \\
\text { Fitted with: }\end{array}$ & $\begin{array}{l}1 \\
\text { S-ManOTBA } \\
\text { NMR } \\
\text { WinEQNMR }\end{array}$ & \\
\hline No. & $c(\mathrm{H})$ & $c(G)$ & $\mathrm{d}(\mathrm{ppm})$ \\
\hline 1 & 1.71E-002 & 1.00E-007 & 9.335 \\
\hline 2 & 1.71E-002 & 4.75E-003 & 9.532 \\
\hline 3 & 1.71E-002 & $9.40 \mathrm{E}-003$ & 9.760 \\
\hline 4 & 1.71E-002 & 1.40E-002 & 9.952 \\
\hline 5 & 1.71E-002 & 1.84E-002 & 10.174 \\
\hline 6 & 1.71E-002 & $2.28 \mathrm{E}-002$ & 10.311 \\
\hline 7 & 1.71E-002 & $2.71 \mathrm{E}-002$ & 10.483 \\
\hline 8 & 1.71E-002 & $3.14 \mathrm{E}-002$ & 10.616 \\
\hline 9 & 1.71E-002 & $3.55 \mathrm{E}-002$ & 10.717 \\
\hline 10 & 1.71E-002 & 3.96E-002 & 10.800 \\
\hline 11 & 1.71E-002 & 4.36E-002 & 10.894 \\
\hline 12 & 1.71E-002 & $4.75 \mathrm{E}-002$ & 10.943 \\
\hline 13 & 1.71E-002 & $5.14 \mathrm{E}-002$ & 11.008 \\
\hline 14 & 1.71E-002 & 5.52E-002 & 11.062 \\
\hline 15 & 1.71E-002 & 5.89E-002 & 11.089 \\
\hline 16 & 1.71E-002 & $6.26 \mathrm{E}-002$ & 11.128 \\
\hline 17 & 1.71E-002 & $6.62 \mathrm{E}-002$ & 11.144 \\
\hline 18 & 1.71E-002 & 7.32E-002 & 11.196 \\
\hline 19 & 1.71E-002 & 7.99E-002 & 11.228 \\
\hline 20 & 1.71E-002 & $9.28 \mathrm{E}-002$ & 11.227 \\
\hline 21 & 1.71E-002 & 1.05E-001 & 11.297 \\
\hline 22 & 1.71E-002 & 1.16E-001 & 11.314 \\
\hline 23 & 1.71E-002 & 1.27E-001 & 11.343 \\
\hline 24 & 1.71E-002 & $1.51 \mathrm{E}-001$ & 11.362 \\
\hline
\end{tabular}


Host: $\quad 3$

Guest: $\quad$ S-ManOTBA

Method: NMR

Fitted with: HypNMR

\begin{tabular}{|c|r|r|r|r|r|r|r|}
\hline No. & $\mathrm{c}(\mathrm{H})$ & $\mathrm{c}(\mathrm{G})$ & $\mathrm{d}$ (ind) & $\mathrm{d}$ (urea) & $\mathrm{d}(\mathrm{C} 9)$ & $\mathrm{d}(\mathrm{C} 10)$ \\
\hline 1 & $1.00 \mathrm{E}-02$ & $0.00 \mathrm{E}+00$ & 8.855 & 8.721 & 8.427 & 8.538 \\
\hline 2 & $9.90 \mathrm{E}-03$ & $1.49 \mathrm{E}-03$ & 8.909 & 9.013 & 8.506 & 8.524 \\
\hline 3 & $9.80 \mathrm{E}-03$ & $2.94 \mathrm{E}-03$ & 8.971 & 9.386 & 8.603 & 8.507 \\
\hline 4 & $9.71 \mathrm{E}-03$ & $4.37 \mathrm{E}-03$ & 9.039 & 9.772 & 8.703 & 8.489 \\
\hline 5 & $9.62 \mathrm{E}-03$ & $5.77 \mathrm{E}-03$ & 9.104 & 10.132 & 8.804 & 8.477 \\
\hline 6 & $9.52 \mathrm{E}-03$ & $7.14 \mathrm{E}-03$ & 9.146 & 10.435 & 8.886 & 8.468 \\
\hline 7 & $9.43 \mathrm{E}-03$ & $8.49 \mathrm{E}-03$ & 9.217 & 10.662 & 8.946 & 8.462 \\
\hline 8 & $9.35 \mathrm{E}-03$ & $9.81 \mathrm{E}-03$ & 9.263 & 10.791 & 8.980 & 8.460 \\
\hline 9 & $9.26 \mathrm{E}-03$ & $1.11 \mathrm{E}-02$ & 9.309 & 10.863 & 8.994 & 8.459 \\
\hline 10 & $9.09 \mathrm{E}-03$ & $1.36 \mathrm{E}-02$ & 9.399 & 10.927 & 9.007 & 8.457 \\
\hline 11 & $8.93 \mathrm{E}-03$ & $1.61 \mathrm{E}-02$ & 9.478 & 10.961 & 9.009 & 8.455 \\
\hline 12 & $8.62 \mathrm{E}-03$ & $2.07 \mathrm{E}-02$ & 9.624 & 10.997 & 9.004 & 8.448 \\
\hline 13 & $8.33 \mathrm{E}-03$ & $2.50 \mathrm{E}-02$ & 9.754 & 11.020 & 8.996 & 8.443 \\
\hline 14 & $8.00 \mathrm{E}-03$ & $3.00 \mathrm{E}-02$ & 9.873 & 11.029 & 8.977 & 8.432 \\
\hline 15 & $7.69 \mathrm{E}-03$ & $3.46 \mathrm{E}-02$ & 9.984 & 11.052 & 8.969 & 8.411 \\
\hline
\end{tabular}

Host: $\quad 4$

Guest: S-ManOTBA

Method: NMR

Fitted with: HypNMR

\begin{tabular}{|c|l|l|r|}
\hline No. & $\mathrm{c}(\mathrm{H})$ & $\mathrm{c}(\mathrm{G})$ & $\mathrm{d}$ (urea) \\
\hline 1 & $5.00 \mathrm{E}-04$ & $0.00 \mathrm{E}+00$ & 8.3333 \\
\hline 2 & $4.95 \mathrm{E}-04$ & $4.95 \mathrm{E}-04$ & 8.4429 \\
\hline 3 & $4.90 \mathrm{E}-04$ & $9.80 \mathrm{E}-04$ & 8.5354 \\
\hline 4 & $4.85 \mathrm{E}-04$ & $1.46 \mathrm{E}-03$ & 8.6211 \\
\hline 5 & $4.81 \mathrm{E}-04$ & $1.92 \mathrm{E}-03$ & 8.6895 \\
\hline 6 & $4.76 \mathrm{E}-04$ & $2.38 \mathrm{E}-03$ & 8.7560 \\
\hline 7 & $4.67 \mathrm{E}-04$ & $3.27 \mathrm{E}-03$ & 8.8521 \\
\hline 8 & $4.59 \mathrm{E}-04$ & $4.13 \mathrm{E}-03$ & 8.9382 \\
\hline 9 & $4.50 \mathrm{E}-04$ & $4.95 \mathrm{E}-03$ & 9.0120 \\
\hline 10 & $4.39 \mathrm{E}-04$ & $6.14 \mathrm{E}-03$ & 9.0908 \\
\hline 11 & $4.27 \mathrm{E}-04$ & $7.26 \mathrm{E}-03$ & 9.1587 \\
\hline 12 & $4.17 \mathrm{E}-04$ & $8.33 \mathrm{E}-03$ & 9.2238 \\
\hline 13 & $4.07 \mathrm{E}-04$ & $9.35 \mathrm{E}-03$ & 9.2793 \\
\hline 14 & $3.97 \mathrm{E}-04$ & $1.03 \mathrm{E}-02$ & 9.3246 \\
\hline 15 & $3.85 \mathrm{E}-04$ & $1.15 \mathrm{E}-02$ & 9.3807 \\
\hline 16 & $3.68 \mathrm{E}-04$ & $1.32 \mathrm{E}-02$ & 9.4396 \\
\hline
\end{tabular}


Host: $\quad 6$

Guest: D-PheOTBA

Method: NMR

\begin{tabular}{|c|c|c|r|r|}
\hline No. & $c(\mathrm{H})$ & $\mathrm{c}(\mathrm{G})$ & $\mathrm{d}(\mathrm{a})$ & $\mathrm{d}(\mathrm{b})$ \\
\hline 1 & $5.00 \mathrm{E}-03$ & $0.00 \mathrm{E}+00$ & 9.1713 & 6.1026 \\
\hline 2 & $5.00 \mathrm{E}-03$ & $7.00 \mathrm{E}-04$ & 9.1609 & 6.0814 \\
\hline 3 & $5.00 \mathrm{E}-03$ & $1.40 \mathrm{E}-03$ & 9.1499 & 6.0639 \\
\hline 4 & $5.00 \mathrm{E}-03$ & $2.10 \mathrm{E}-03$ & 9.1412 & 6.0474 \\
\hline 5 & $5.00 \mathrm{E}-03$ & $2.80 \mathrm{E}-03$ & 9.1336 & 6.0327 \\
\hline 6 & $5.00 \mathrm{E}-03$ & $3.50 \mathrm{E}-03$ & 9.1301 & 6.0294 \\
\hline 7 & $5.00 \mathrm{E}-03$ & $4.20 \mathrm{E}-03$ & 9.1252 & 6.2280 \\
\hline 8 & $5.00 \mathrm{E}-03$ & $4.90 \mathrm{E}-03$ & 9.1244 & 6.0199 \\
\hline 9 & $5.00 \mathrm{E}-03$ & $5.60 \mathrm{E}-03$ & 9.1200 & 6.0193 \\
\hline 10 & $5.00 \mathrm{E}-03$ & $6.30 \mathrm{E}-03$ & 9.1207 & 6.0194 \\
\hline 11 & $5.00 \mathrm{E}-03$ & $7.00 \mathrm{E}-03$ & 9.1213 & 6.0223 \\
\hline 12 & $5.00 \mathrm{E}-03$ & $7.88 \mathrm{E}-03$ & 9.1247 & 6.0269 \\
\hline 13 & $5.00 \mathrm{E}-03$ & $8.75 \mathrm{E}-03$ & 9.1286 & 6.0318 \\
\hline 14 & $5.00 \mathrm{E}-03$ & $9.63 \mathrm{E}-03$ & 9.1311 & 6.0351 \\
\hline 15 & $5.00 \mathrm{E}-03$ & $1.05 \mathrm{E}-02$ & 9.1324 & 6.0366 \\
\hline 16 & $5.00 \mathrm{E}-03$ & $1.23 \mathrm{E}-02$ & 9.1331 & 6.0374 \\
\hline 17 & $5.00 \mathrm{E}-03$ & $1.40 \mathrm{E}-02$ & 9.1330 & 6.0373 \\
\hline 18 & $5.00 \mathrm{E}-03$ & $1.58 \mathrm{E}-02$ & 9.1326 & 6.0367 \\
\hline 19 & $5.00 \mathrm{E}-03$ & $1.75 \mathrm{E}-02$ & 9.1323 & 6.0362 \\
\hline 20 & $5.00 \mathrm{E}-03$ & $1.93 \mathrm{E}-02$ & 9.1321 & 6.0358 \\
\hline 21 & $5.00 \mathrm{E}-03$ & $2.10 \mathrm{E}-02$ & 9.1314 & 6.0354 \\
\hline
\end{tabular}


Host: $\quad 6$

Guest: D-PheOTBA

Method: UV-Vis

Fitted with: HypSpec

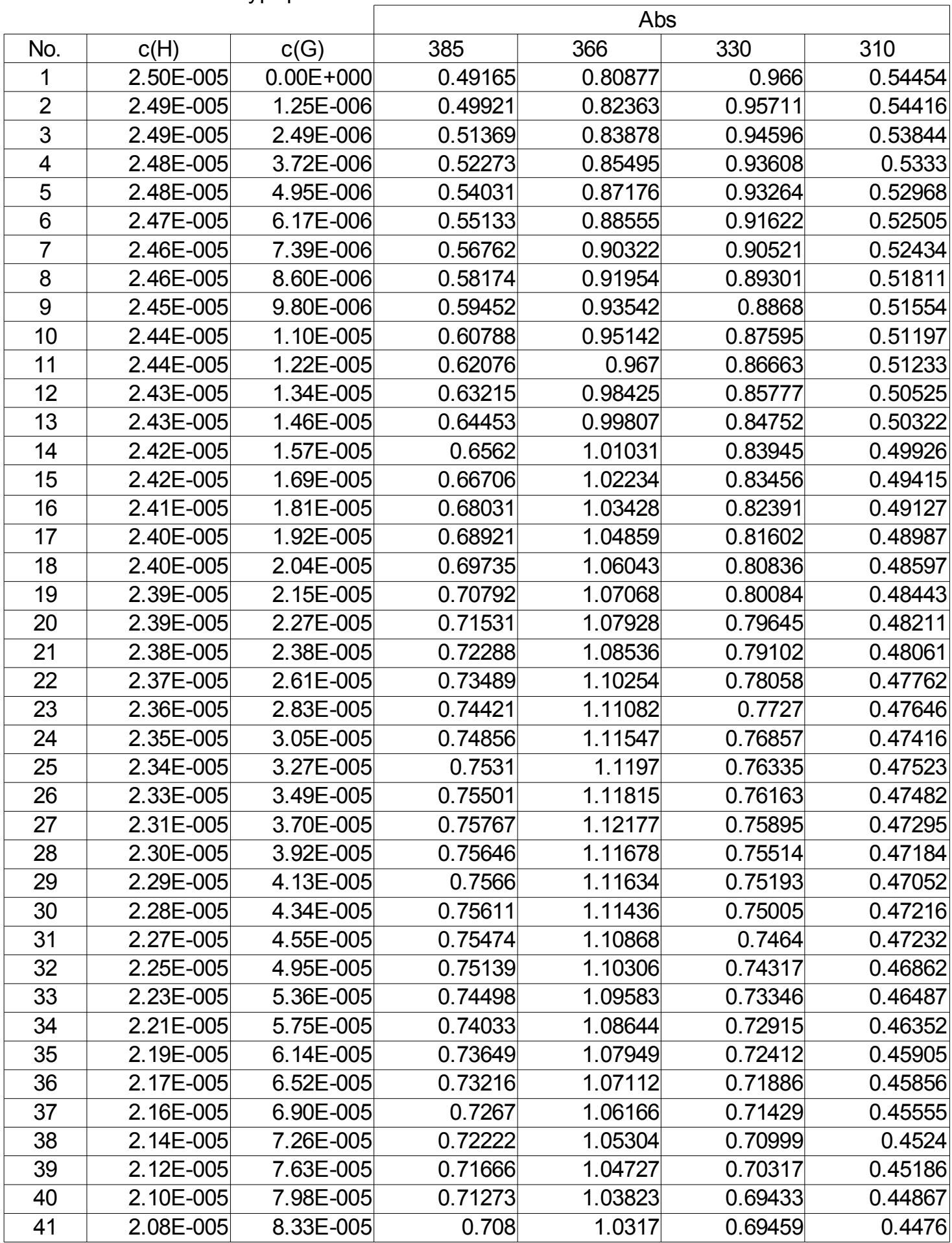

